\title{
Clinical features that affect the number of pelvic lymph nodes harvested in patients with cervical cancer stage IB1 to IIA2
}

Yae Ji Choi, MD¹, Woo Yeon Hwang, MD', Nara Lee, MD², Miseon Kim, MD², Dong Hoon Suh, MD, PhD', Kidong Kim, MD, PhD'1, Yong Beom Kim, MD, PhD', Jae Hong No, MD, PhD

Department of Obstetrics and Gynecology, ${ }^{1}$ Seoul National University Bundang Hospital, Seongnam, ${ }^{2} \mathrm{CHA}$ Gangnam Medical Center, CHA University School of Medicine, Seoul, Korea

Objective

To investigate clinical features that affect the number of pelvic lymph nodes (PLNs) harvested and prognostic significance of the number of PLNs removed in patients with stage IB1 to IIA2 cervical cancer.

\section{Methods}

Data from patients with cervical cancer whom underwent hysterectomy with PLN dissection between June 2004 and July 2015 were reviewed retrospectively. Data on clinicopathologic factors including age, height, and weight were collected. Data on the presence of PLN metastasis on imaging studies prior to surgery, number of PLNs harvested, and presence of metastasis in the harvested PLNs were retrieved from medical records. Clinical features associated with the number of PLNs harvested were analyzed. Disease-free survival (DFS) and overall survival (OS) according to the number of PLNs harvested were analyzed.

Results

During the study period, 210 patients were included. The height and weight of patients and preoperative positive positron emission tomography findings were significantly associated with a higher number of PLNs harvested. As a pathologic factor, larger tumor size was associated with a higher number of PLNs harvested. Furthermore, a higher number of PLNs harvested was associated with a higher number of metastatic PLNs and patients undergoing postoperative concurrent chemoradiation therapy. Patient height and tumor size were independent factors affecting the number of PLNs harvested in multivariate analysis. However, the number of PLNs harvested was not associated with DFS or OS.

\section{Conclusion}

The number of PLNs harvested during surgery was associated with patient height; however, this was not related to the prognosis of the disease.

Keywords: Uterine cervical neoplasms; Lymph nodes; Lymph node excision

\section{Introduction}

Cervical cancer is the second leading cause of cancer-related deaths in women aged 20 to 39 years, and approximately 9 women with cervical cancer in this age group die every week worldwide [1]. In Korea, even though the incidence of cervical cancer is decreasing steadily, it is still the third leading cause of cancer-related deaths among Korean women [2].
Received: 2020.01.07. Revised: 2020.07.21. Accepted: 2020.09.15.

Corresponding author: Jae Hong No, MD, PhD

Department of Obstetrics and Gynecology, Seoul National

University Bundang Hospital, 82 Gumi-ro 173-beon-gil, Bundanggu, Seongnam 13620, Korea

E-mail: jhno@snu.ac.kr

https://orcid.org/0000-0002-2389-6757

Dong Hoon Suh has been an Editorial Board of Obstetrics \& Gynecology Science; however, he was not involved in the peer reviewer selection, evaluation, or decision process of this article. Otherwise, no other potential conflicts of interest relevant to this article was reported.

Articles published in Obstet Gynecol Sci are open-access, distributed under the terms of the Creative Commons Attribution Non-Commercial License (http://creativecommons. org/licenses/by-nc/3.0/) which permits unrestricted non-commercial use, distribution, and reproduction in any medium, provided the original work is properly cited.

Copyright $\odot 2021$ Korean Society of Obstetrics and Gynecology 


\title{
Obstetrics \& Gynecology Science
}

\author{
Vol. 64, No. 1, 2021
}

Therefore, improving the prognosis of the disease remains essential. Surgery is the treatment of choice, and radical hysterectomy with pelvic lymphadenectomy is the standard surgical method for patients with early-stage cervical cancer. A less aggressive surgical approach is applied to treat microinvasive cervical cancer (2014 International Federation of Gynecology and Obstetrics [FIGO] stage IA1 to IA2), whereas a more radical approach is used to treat invasive cancers (FIGO stage IB1 to IIA2). Lymph node (LN) involvement is one of the strongest prognostic factors for decreased survival in women with cervical cancer undergoing radical surgery [3]; therefore, the presence of $L N$ metastasis has recently been included in the 2018 FIGO staging system [4]. Although complete uniform pelvic lymphadenectomy is the routine procedure, the total number of pelvic LNs (PLNs) harvested varies among patients. Lymphatic dissemination to the regional LNs has been suggested as the main route of metastasis in cervical cancer [5]. Therefore, complete removal of PLNs is essential to improve survival, as it may increase the likelihood of detecting occult metastatic lesions [6]. Theoretically, it is possible that patients with a larger number of PLNs may have additional routes for lymphatic dissemination. Therefore, this retrospective study aimed to determine the clinical features that affect the number of PLNs harvested, and the prognostic significance of the total number of PLNs removed in patients with cervical cancer stage IB1 to IIA2, whom underwent radical hysterectomy with PLN dissection.

\section{Materials and methods}

\section{Case selection}

Clinical data from 215 consecutive patients with cervical cancer stage IB1 to IIA2 whom underwent radical hysterectomy with PLN dissection, without receiving preoperative therapy, between June 2004 and July 2015, were reviewed retrospectively. For the staging of cervical cancer, the 2014 FIGO staging system was implemented. The inclusion criteria were as follows: 1) patients with FIGO stage IB1 to IIA2 cervical cancer; 2) patients whom underwent primary surgery, including radical hysterectomy with PLN dissection; 3) no history of surgery, radiotherapy, or chemotherapy for cervical cancer, with the exception of large loop excision of the transformation zone for clinical staging; and 4) those without underlying medical history that would affect survival. From a total of
215 patients, 5 were not eligible and excluded. Therefore, a total of 210 patients were included for the statistical analysis. The study received Institutional Review Board approval (B-1903-529-102), and the need for informed consent was waived as the current study was conducted as a retrospective review.

\section{Data collection}

Data on clinicopathologic factors including age, body weight, and height were collected. The presence of PLN metastasis on imaging studies such as pelvic computed tomography (CT), magnetic resonance imaging (MRI), and positron emission tomography (PET) prior to surgery was reviewed by radiologists.

\section{Surgical procedure}

All patients underwent radical hysterectomy (type $C$ of the Querleu and Morrow surgical classification system), with or without bilateral salpingo-oophorectomy. Additionally, bilateral PLN dissection with or without para-aortic LN dissection was performed in all cases. PLNs were harvested from nodebearing fat tissue. All surgeries were performed via laparotomy or laparoscopy, and the method of approach was decided by the surgeon's preference and patient characteristics.

\section{Pathologic specimen review}

Pathologic evaluation included the number of PLNs harvested, histology, tumor size, presence of lymphovascular space invasion (LVSI), and presence of PLN metastasis. The patients were divided into 2 groups according to the mean number (23) of PLNs harvested.

\section{Adjuvant therapy and follow-up}

Postoperative adjuvant treatment was administered to patients with more than 2 intermediate-risk factors for recurrence according to the Sedlis criteria: 1) large tumor size $>4$ $\mathrm{cm} ; 2)$ cervical stromal invasion into the middle or deep onethird; and 3) LVSI [7], and to those who had one of the highrisk factors: 1) positive or close margins; 2) positive LNs; and 3) microscopic parametrial involvement [8]. All patients underwent concurrent chemoradiation therapy with platinumbased chemotherapy. Data of post-treatment follow-up of all patients were retrospectively reviewed, including their serum tumor markers and imaging exams (CT, MRI, or PET). Disease-free survival (DFS) was defined as the period be- 


\section{Obstetrics \& Gynecology Science}

Yae Ji Choi, et al. Pelvic lymph nodes in cervical cancer

tween the date of primary surgery and the date of clinically proven recurrence. The overall survival (OS) was defined as the period between the date of primary surgery and the date of cancer-related death.

\section{Statistical analyses}

The association between clinical features, including the number of harvested PLNs and survival outcome of cervical cancer, was determined using the Kaplan-Meier method with the log-rank test and multivariate Cox proportional hazard analysis with odds ratio (OR) and 95\% confidence interval (CI). Pearson's $\chi^{2}$ test or the Student's $t$-test was used to compare characteristics. Statistical analysis was performed using the Statistical Package for Social Sciences version 17.0 (IBM Corp., Armonk, NY, USA); a P-value $<0.05$ was considered significant.

\section{Results}

A total of 210 patients who met the inclusion criteria were enrolled. The clinicopathological characteristics are shown in Table 1. Age was divided into 2 groups: $<50$ years (114 patients, $54.3 \%$ ) and $\geq 50$ years (96 patients, $45.7 \%$ ). The body weights of the patients were divided into 2 groups: $<60 \mathrm{~kg}$ (142 patients, $67.6 \%$ ) and $\geq 60 \mathrm{~kg}$ (68 patients, $32.4 \%$ ). The cutoff value for the height of patients for predicting PLN number, $<159.6 \mathrm{~cm}$ (130 patients, $61.9 \%$ ) and $\geq 159.6 \mathrm{~cm}$ (80 patients, $38.1 \%)$, was calculated using the receiver operating characteristic curve. The pathological types were identified as squamous cell carcinoma (SCC) (120 cases, 57.1\%) and non-SCC (90 cases, 42.9\%). Regarding the FIGO stage of the patients, type IB1 was the most common, equaling 148 cases $(70.5 \%) ; 46$ cases were IB2 $(21.9 \%), 12$ cases were IIA1 (5.7\%), and 4 cases were IIA2 (1.9\%). Sixty patients had a tumor size $\geq 4 \mathrm{~cm}(28.6 \%)$ and 150 cases had a tumor size $<4 \mathrm{~cm}(71.4 \%)$. LVSI was present in 100 cases (47.6\%). Metastatic PLN was observed in 42 (20.0\%) cases, and 168 cases $(80.0 \%)$ had no PLN metastasis. Concurrent chemoradiation therapy as an adjuvant treatment was administered to 92 patients (43.8\%), whereas 118 patients (56.2\%) were not treated post-surgically.

The mean number of total PLNs harvested was $23.1 \pm 11.7$ and the highest number of PLNs harvested in one case was 65. Patients were classified into 2 groups according to the
Table 1. Clinicopathologic characteristics of the patients

\begin{tabular}{lcc}
\hline Variables & $\begin{array}{c}\text { No. of patients } \\
(\mathbf{n}=\mathbf{2 1 0})\end{array}$ & Value \\
\hline Age & & \\
Mean & $47(25-81)$ & \\
$<50$ & 114 & 54.3 \\
$\geq 50$ & 96 & 45.7 \\
Body weight $(\mathrm{kg})$ & & \\
Mean & $57.6 \pm 8.17$ & \\
$<60$ & 142 & 67.6 \\
$\geq 60$ & 68 & 32.4
\end{tabular}

Height (cm)

$\begin{array}{ccc}\text { Mean } & 157.3 \pm 6.68 & \\ <159.6 & 130 & 61.9 \\ \geq 159.6 & 80 & 38.1\end{array}$

Body mass index $\left(\mathrm{kg} / \mathrm{m}^{2}\right)$

$\begin{array}{lcc}\text { Mean } & 23.34 \pm 3.54 & \\ <25 & 152 & 72.4 \\ \geq 25 & 58 & 27.6\end{array}$

FIGO clinical stage

$\begin{array}{lcc}\text { IB1 } & 148 & 70.5 \\ \mid \mathrm{IB} 2 & 46 & 21.9 \\ \| \mathrm{I} 1 & 12 & 5.7 \\ \| \mathrm{A} 2 & 4 & 1.9\end{array}$

Histology

$\begin{array}{lll}\text { Non-SCC } & 90 & 42.9\end{array}$

SCC $\quad 120 \quad 57.1$

Tumor size $(\mathrm{cm})$

$\begin{array}{lll}<4 & 150 & 71.4\end{array}$

$\begin{array}{lll}\geq 4 & 60 & 28.6\end{array}$

LVSI

No

$110 \quad 52.4$

$\begin{array}{lll}\text { Yes } & 100 & 47.6\end{array}$

Harvested PLN

$\begin{array}{lcl}\text { Mean } & 23.13 \pm 11.66 & \\ <23 & 110 & 52.4 \\ \geq 23 & 100 & 47.6\end{array}$

PLN metastasis

$\begin{array}{lll}\text { No } & 168 & 80.0\end{array}$

$\begin{array}{lll}\text { Yes } & 42 & 20.0\end{array}$

Adjuvant CCRT

$\begin{array}{lll}\text { No } & 118 & 56.2\end{array}$

$\begin{array}{lll}\text { Yes } & 92 & 43.8\end{array}$

Value are shown as mean \pm standard deviation or number (\%).

FIGO, International Federation of Gynecology and Obstetrics; SCC, squamous cellular carcinoma; LVSI, lymphovascular space invasion; PLN, pelvic lymph node; CCRT, concurrent chemo-radiation therapy. 


\title{
Obstetrics \& Gynecology Science
}

\author{
Vol. 64, No. 1, 2021
}

number of harvested PLNs as shown in Table 2; 110 cases (52.4\%) had <23 PLNs harvested, and 100 cases (47.6\%) had $\geq 23$ PLNs harvested. Data were analyzed to determine the association between the number of PLNs harvested and other clinicopathologic factors. The results showed that greater patient body weight $(\mathrm{OR}, 1.952 ; 95 \% \mathrm{Cl}, 1.086-$ $3.510 ; P=0.024)$ and height $(O R, 1.753 ; 95 \% \mathrm{Cl}, 1.000$ 3.076; $P=0.049$ ) were associated with a higher number of

Table 2. Clinicopathologic factors affecting the number of harvested pelvic lymph nodes (PLNs)

\begin{tabular}{|c|c|c|c|c|c|c|}
\hline \multirow{2}{*}{ Variables } & & \multicolumn{2}{|c|}{ Harvested PLN } & \multirow{2}{*}{ OR } & \multirow{2}{*}{$95 \% \mathrm{Cl}$} & \multirow{2}{*}{$P$-value } \\
\hline & & $<23$ & $\geq 23$ & & & \\
\hline \multirow[t]{2}{*}{ Age } & $<50$ & 58 & 56 & 0.876 & $0.509-1.510$ & 0.634 \\
\hline & $\geq 50$ & 52 & 44 & & & \\
\hline \multirow[t]{2}{*}{$\mathrm{BMI}\left(\mathrm{kg} / \mathrm{m}^{2}\right)$} & $<25$ & 82 & 70 & 1.255 & $0.685-2.300$ & 0.462 \\
\hline & $\geq 25$ & 28 & 30 & & & \\
\hline \multirow[t]{2}{*}{ Body weight (kg) } & $<60$ & 82 & 60 & 1.952 & $1.086-3.510$ & 0.024 \\
\hline & $\geq 60$ & 28 & 40 & & & \\
\hline \multirow[t]{2}{*}{ Height (cm) } & $<159.6$ & 75 & 55 & 1.753 & $1.000-3.076$ & 0.049 \\
\hline & $\geq 159.6$ & 35 & 45 & & & \\
\hline \multirow[t]{2}{*}{ CCRT } & No & 70 & 48 & 1.896 & $1.092-3.293$ & 0.023 \\
\hline & Yes & 40 & 52 & & & \\
\hline \multirow[t]{2}{*}{ LN metastasis on MRI } & No & 66 & 48 & 1.594 & $0.921-2.758$ & 0.095 \\
\hline & Yes & 44 & 51 & & & \\
\hline \multirow[t]{2}{*}{ LN metastasis on PET } & No & 71 & 33 & 2.613 & $1.152-5.926$ & 0.019 \\
\hline & Yes & 14 & 17 & & & \\
\hline \multirow[t]{2}{*}{ LN metastasis on CT } & No & 63 & 46 & 1.574 & $0.912-2.715$ & 0.102 \\
\hline & Yes & 47 & 54 & & & \\
\hline \multirow[t]{2}{*}{ Tumor size $(\mathrm{cm})$} & $<4$ & 91 & 59 & 3.328 & $1.764-6.280$ & $<0.001$ \\
\hline & $\geq 4$ & 19 & 41 & & & \\
\hline \multirow[t]{2}{*}{ LVSI } & No & 59 & 51 & 1.111 & $0.646-1.912$ & 0.702 \\
\hline & Yes & 51 & 49 & & & \\
\hline \multirow[t]{2}{*}{ Operation method } & Laparotomy & 40 & 74 & 0.201 & $0.111-0.363$ & $<0.001$ \\
\hline & Laproscopy & 70 & 26 & & & \\
\hline \multirow[t]{2}{*}{ Pathologically confirmed metastasis } & No & 96 & 72 & 2.667 & $1.310-5.427$ & 0.006 \\
\hline & Yes & 14 & 28 & & & \\
\hline
\end{tabular}

OR, odds ratio; $\mathrm{Cl}$, confidence interval; BMI, body mass index; CCRT, concurrent chemo-radiation therapy; LN, lymph node; MRI, magnetic resonance imaging; PET, positron emission tomography; CT, computed tomography; LVSI, lymphovascular space invasion.

Table 3. Multivariate logistic regression analysis of independent factors affecting the number of harvested pelvic lymph nodes

\begin{tabular}{lccccc}
\hline Variables & Beta & SE & OR & 95\% Cl & P-value \\
\hline Body weight $(\geq 60 \mathrm{~kg})$ & 0.485 & 0.437 & 1.624 & $0.690-3.823$ & 0.267 \\
Height $(\geq 159.6 \mathrm{~cm})$ & 0.910 & 0.422 & 2.484 & $1.086-5.682$ & 0.031 \\
LN metastasis on MRI & 0.496 & 0.423 & 1.642 & $0.716-3.765$ & 0.241 \\
LN metastasis on PET & 0.180 & 0.531 & 1.198 & $0.423-3.395$ & 0.734 \\
Tumor size $(\geq 4 \mathrm{~cm})$ & 1.516 & 0.555 & 4.555 & $1.536-13.506$ & 0.006 \\
Surgery method & -0.360 & 0.487 & 0.698 & $0.268-1.813$ & 0.460 \\
\hline
\end{tabular}

SE, standard error; OR, odds ratio; Cl, confidence interval, LN, lymph node; MRI, magnetic resonance imaging; PET, positron emission tomography. 


\section{Obstetrics \& Gynecology Science}

Yae Ji Choi, et al. Pelvic lymph nodes in cervical cancer

PLNs harvested. In addition, the presence of PLN metastasis on preoperative PET scans (OR, 2.613; 95\% Cl, 1.152-5.926; $P=0.019$ ) was further associated with a higher number of total PLNs harvested. There was no significant difference in patient age $(O R, 0.876 ; 95 \% \mathrm{Cl}, 0.509-1.510 ; P=0.634)$, preoperative MRI (OR, 1.594; 95\% Cl, 0.921-2.715; $P=0.095)$, and $\mathrm{CT}(\mathrm{OR}, 1.574 ; 95 \% \mathrm{Cl}, 0.912-2.715 ; P=0.102)$ studies in association with the number of PLNs harvested. A higher number of PLNs harvested was additionally associated with larger tumor size $\geq 4 \mathrm{~cm}(\mathrm{OR}, 3.328 ; 95 \% \mathrm{Cl}, 1.764-6.280$; $P<0.001)$ and pathologically confirmed PLN metastasis (OR, $2.667 ; 95 \% \mathrm{Cl}, 1.31-5.427 ; P=0.006)$, but not with the pres-

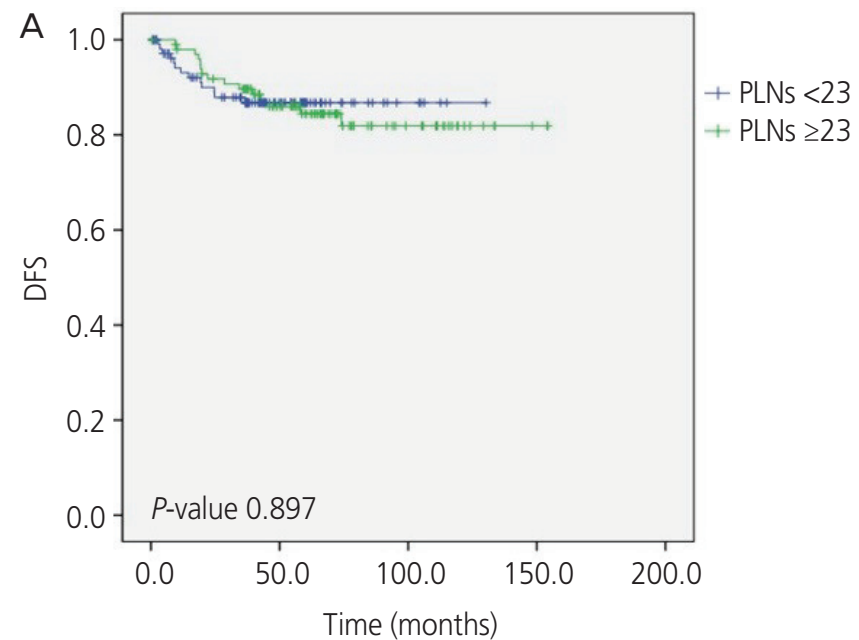

ence of LVSI (OR, 1.111; 95\% Cl, 0.646-1.912; $P=0.702)$. Regarding the operation method, a higher number of PLNs harvested was associated with the laparotomy approach compared to laparoscopy $(\mathrm{OR}, 0.201 ; 95 \% \mathrm{Cl}, 0.111-0.363$; $P<0.001)$.

A multivariate logistic regression analysis was carried out to investigate which factors were independently associated with a higher number of PLNs harvested, as shown in Table 3. The results showed that only greater height $(\mathrm{OR}, 2.484 ; 95 \% \mathrm{Cl}$, 1.086-5.682; $P=0.031)$ and tumor size $\geq 4 \mathrm{~cm}(O R, 4.555$; $95 \% \mathrm{Cl}, 1.536-13.506 ; P=0.006)$ were associated with a higher number of PLNs harvested, whereas body weight

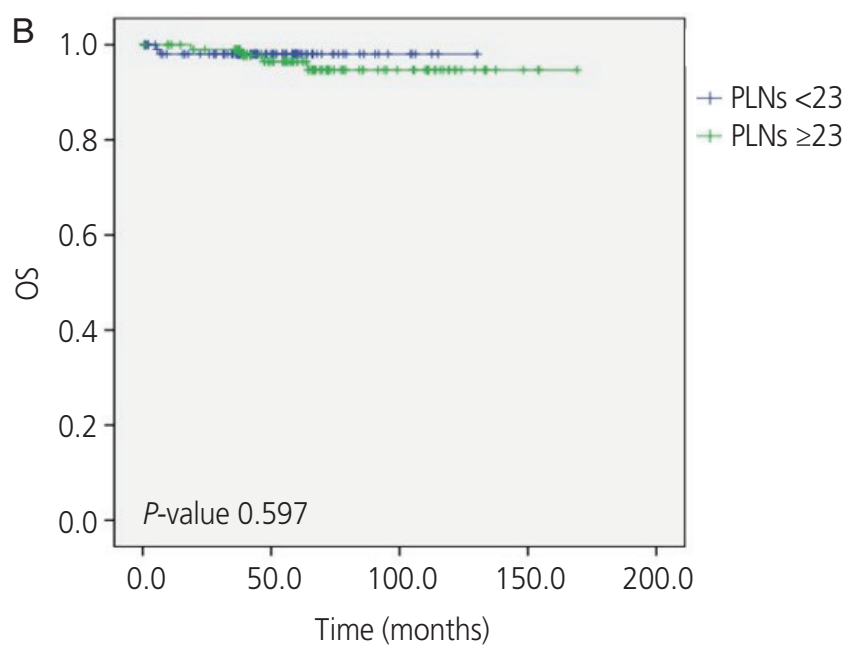

Fig. 1. Kaplan-Meier analysis of (A) disease free survival (DFS) and (B) overall survival (OS) post-surgery of both groups with a cut-off based on the average number of pelvic lymph nodes (PLNs) harvested.
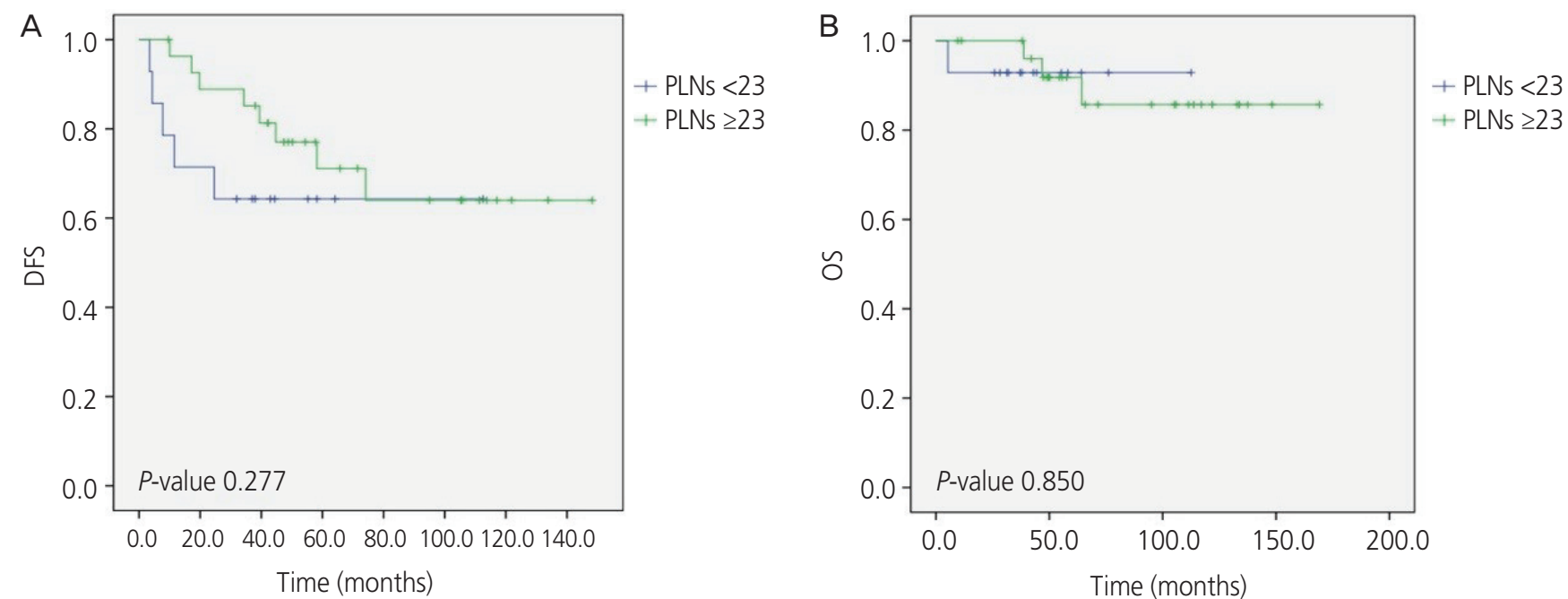

Fig. 2. Kaplan-Meier analysis of (A) disease free survival (DFS) and (B) overall survival (OS) post-surgery in the pelvic lymph node (PLN) positive patients of both groups with a cut-off based on the average number of PLNs harvested. 


\title{
Obstetrics \& Gynecology Science
}

\author{
Vol. 64, No. 1, 2021
}

(OR, 1.624; 95\% Cl, 0.690-3.823; $P=0.267)$ and preoperative LN metastasis present on PET scans (OR, 1.198; $95 \% \mathrm{Cl}$, 0.423-3.395; $P=0.734$ ) showed no significant associations. In addition, the operation method showed no association in the multivariate analysis $(\mathrm{OR}, 0.698 ; 95 \% \mathrm{Cl}, 0.268-1.813$; $P=0.460$ ).

The 5 -year DFS was $87.1 \%$ overall, $86.0 \%$ for the $\geq 23$ PLNs harvested group and $88.2 \%$ for the $<23$ PLNs harvested group. The 5-year OS was $97.1 \%$ overall, $96.0 \%$ for the $\geq 23$ PLNs harvested group and $98.2 \%$ for the $<23$ PLNs harvested group. The number of PLNs harvested had no significant influence on prognosis ( $D F S, P=0.897 ; O S, P=0.597$ ) of the disease, as shown in the Kaplan-Meier graph in Fig. 1. Additionally, subgroup analysis within the pathologically confirmed metastasis group was carried out, which showed no significant difference in DFS $(P=0.277)$ or OS $(P=0.850)$, as shown in Fig. 2 .

\section{Discussion}

Up to now, a lot of research regarding the prognostic value of pelvic lymphadenectomy and the total number of PLNs in patients with early-stage cervical cancer has been carried out. However, there have been no studies that have focused on the total number of PLNs in each patient or which clinicopathologic factors affect the total number regardless of the presence of PLN metastasis. In this retrospective study, characteristics associated with a higher number of total PLNs harvested were patient height and weight. This could be due to the simple assumption that more PLNs are present in larger patients. Another finding of this study was that a higher number of PLNs harvested was associated with the laparotomy approach compared to laparoscopy. The surgical method may affect the surgeon's performance, as some surgeons found laparoscopic PLN retrieval more difficult. The results of this study showed that a larger tumor size $(\geq 4 \mathrm{~cm})$ and the presence of PLN metastasis on preoperative PET findings were further associated with a higher number of total PLNs retrieved in these patients. When performing pelvic lymphadenectomy, it is routine to attempt to remove every PLN present; however, it is inevitable that the degree of extensiveness is affected by the surgeon and the difficulty of the surgical procedure. With preoperative findings of larger tumor size and PET images suggesting PLN metastasis, the surgeon may perform pelvic lymphadenectomy more thoroughly, resulting in more PLNs being retrieved during surgery. Interestingly, a higher number of PLNs retrieved during surgery was associated with patients undergoing CCRT and the presence of pathologically confirmed PLN metastasis. This finding may be due to a higher chance of finding occult metastasis with more PLNs removed, thus leading to adjuvant CCRT treatment. When multivariate analysis was performed, the factors significantly associated with a higher number of PLNs in patients with early cervical cancer were patient height and tumor size. Regardless of these findings, the total number of PLNs harvested did not affect DFS or OS in patients with early cervical cancer in this study.

Without a doubt, there has been much controversy regarding whether the number of PLNs removed improves survival in previous studies. Pieterse et al. [9] showed that a higher number of PLNs retrieved was associated with better survival in patients with positive PLNs. In another study by Shah et al. [10] based on the Surveillance Epidemiology and End Results database, it was shown that a higher number of PLNs retrieved had a positive impact on survival in patients without nodal metastasis. In contrast, in a study by Ditto et al., [11] the total number of LNs removed during systematic lymphadenectomy was not a significant prognostic factor for survival. However, none of these studies examined factors that affect the total number of removed PLNs.

In theory, when performing systematic lymphadenectomy, it is essential to completely remove all the PLNs regardless of metastasis status; therefore, the total number of PLNs removed is dependent on the nodal yield of each patient. However, it is further affected by the thoroughness of LN dissection performed by the surgeon, which leads to one of the limitations of this study. All participants were enrolled from a single institution, and the surgeries performed by only 4 surgeons. Although it is inevitable that the thoroughness may vary depending on the surgeon, it should be emphasized that all patients in this study received systematic pelvic lymphadenectomy, and the institution ensured consistent treatment guidelines and surgical skills. Further limitations of this study include the retrospective nature of the study and that the sample size may be considered small to draw definitive conclusions. In addition, sentinel LN detection and sampling was not considered in this study, despite it being accepted as standard practice in many centers for early cervical cancer patients. However, it seems that this is the first study 


\section{Obstetrics \& Gynecology Science}

Yae Ji Choi, et al. Pelvic lymph nodes in cervical cancer

focusing on the total number of PLNs removed dependent on patient specific nodal yield, and which clinicopathologic factors affect the total number of PLNs.

In conclusion, the total number of PLNs harvested during surgery, which may reflect the total nodal yield of each patient, was associated with patient size, especially height. Larger tumor size was associated with a higher number of PLNs retrieved. Recent studies have introduced the importance of the prognostic value of LN ratio; for example, in the study by Aslan et al., [12] it has been proposed that the ratio of positive LNs to the total number of LNs removed is an independent prognostic factor for decreased DFS and OS. Although this study showed that the total number of PLNs harvested did not affect DFS or OS in patients with early cervical cancer, the total number of PLNs was associated with some clinicopathological factors of the patients, such as height.

\section{Conflict of interest}

No potential conflict of interest relevant to this article was reported.

\section{Ethical approval}

The study received Institutional Review Board approval (B1903-529-102).

\section{Patient consent}

Informed consent was waived as the current study was conducted as a retrospective review.

\section{Funding information}

None.

\section{References}

1. Siegel RL, Miller KD, Jemal A. Cancer statistics, 2019. CA Cancer J Clin 2019;69:7-34.
2. Kweon SS. Updates on cancer epidemiology in Korea, 2018. Chonnam Med J 2018;54:90-100.

3. Delgado G, Bundy B, Zaino R, Sevin BU, Creasman WT, Major F. Prospective surgical-pathological study of disease-free interval in patients with stage IB squamous cell carcinoma of the cervix: a Gynecologic Oncology Group study. Gynecol Oncol 1990;38:352-7.

4. Bhatla N, Aoki D, Sharma DN, Sankaranarayanan R. Cancer of the cervix uteri. Int J Gynaecol Obstet 2018;143 Suppl 2:22-36.

5. Huang BX, Fang F. Progress in the study of lymph node metastasis in early-stage cervical cancer. Curr Med Sci 2018;38:567-74.

6. Lim S, Cho K, Lee S, Lee K, Shin J, Chung D, et al. Effect of number of retrieved lymph nodes on prognosis in FIGO stage IB-IIA cervical cancer patients treated with primary radical surgery. J Obstet Gynaecol Res 2017;43:211-9.

7. Peters WA 3rd, Liu PY, Barrett RJ 2nd, Stock RJ, Monk BJ, Berek JS, et al. Concurrent chemotherapy and pelvic radiation therapy compared with pelvic radiation therapy alone as adjuvant therapy after radical surgery in high-risk early-stage cancer of the cervix. J Clin Oncol 2000;18:1606-13.

8. Oaknin A, Rubio MJ, Redondo A, De Juan A, Cueva Bañuelos JF, Gil-Martin M, et al. SEOM guidelines for cervical cancer. Clin Transl Oncol 2015;17:1036-42.

9. Pieterse QD, Kenter GG, Gaarenstroom KN, Peters AA, Willems SM, Fleuren GJ, et al. The number of pelvic lymph nodes in the quality control and prognosis of radical hysterectomy for the treatment of cervical cancer. Eur J Surg Oncol 2007;33:216-21.

10. Shah M, Lewin SN, Deutsch I, Burke WM, Sun X, Herzog $T J$, et al. Therapeutic role of lymphadenectomy for cervical cancer. Cancer 2011;117:310-7.

11. Ditto A, Martinelli F, Lo Vullo S, Reato C, Solima E, Carcangiu $\mathrm{M}$, et al. The role of lymphadenectomy in cervical cancer patients: the significance of the number and the status of lymph nodes removed in 526 cases treated in a single institution. Ann Surg Oncol 2013;20:3948-54.

12. Aslan K, Meydanli MM, Oz M, Tohma YA, Haberal A, Ayhan $A$. The prognostic value of lymph node ratio in stage IIIC cervical cancer patients triaged to primary treatment by radical hysterectomy with systematic pelvic and para-aortic lymphadenectomy. J Gynecol Oncol 2020;31:e1. 